\title{
THEORY OF DOUBLY RADIATIVE $\pi$ CAPTURE IN NUCLEI
}

P. Christillin and T.E.O. Ericson

CERN - Geneva

\section{$A B S T R A C T$}

A detailed theory is developed for the nuclear $\left(\pi^{-}, 2 \gamma\right)$ capture process assuming a normal nucleus with no anomaly due to the meson field. An effective Hamiltonian is constructed from the individual gauge invariant nucleonic process. Branching ratio and angular correlations are calculated using the closure approximation and a realistic shape of the nuclear excitation spectrum.

It is shown that the atomic $1 \mathrm{~s}$ capture is particularly sensitive to pion effects, while the $2 p$ atomic capture is dominated by the bremsitrahlung of single radiative capture, Explicit numerical results are given for ${ }^{12} \mathrm{C}$ and compared with recent experimental work. While the branching ratio agrees, a qualitative discrepancy is found in backward angular shape.

Ref. TH. 2694-CERN

21 June 1979 
1. It has been pointed out [1] that the doubly radiative $\pi$ capture process in nuclei, $\pi^{-}+{ }^{Z} A \rightarrow \gamma_{1}+\gamma_{2}+{ }^{-1} A^{*}$, is potentially a sensitive probe of nuclear pionic degrees of freedom. The reason for this is that under the right kinematicai conditions (zero-momentum pion) the process is dominated by the annihilation of the $\pi^{-}$on a virtual $\pi^{+}$in the nucleus, i.e. $\pi^{-}+" \pi^{+} " \rightarrow \gamma_{1}+\gamma_{2}$. As a consequence a major anomaly in the virtual $\pi^{+}$field of the nucleus will modify the doubly radiative process. Such anomalies could be associated with nuclear pion condensation [2] as discussed in the present context by Barshay [3] or with precursor phenomena to this condensation [4]. This has prompted us to a detailed theoretical investigation of the reaction assuming a conventional nuclear picture. Our purpose is to establish those conditions for which the mesonic contributions are of particular importance. In addition, we wish to establish a good theoretical comparison standard, which can serve to pinpoint major experimental discrepancies due to mesonic effects. There is a particular need for such a quantitative description at this moment, since the process has recently been observed for the first time [5] with information both on the rate and on the angular correlations. Inside a standard nuclear picture a magnitude estimate of the $2 \gamma$ reaction has previously been given by Ericson and Wilkin [1] for the pion in the atomic 1s-state. The estimate is based on the ratio of the double to the single radiative capture on the proton, with the argument that this ratio should be roughly the same in a nucleus, since the momentum transfer is similar.

As concerns the nucleonic process we refer the reader to the recent study by Beder [6]. He gives the explicit relativistic and non-relativistic amplitude and discusses in detail the relative importance of the different diagrams for low momentum pions in various kinematical conditions. We have independently obtained the identical amplitude.

Our present approach to the nuclear process is based on an effective Hamiltonian description. We will therefore assume that the process takes place only on individual nucleons and with the individual nucleon capture amplitude. 
The effective Hamiltonian is then constructed in close analogy to those used in $\mu$ capture and radiative $\pi$ capture. In the same spirit only the leading nonrelativistic terms are kept in the nucleon amplitude, but we take explicitly into account that the pion may be either in a relative $\pi N$ s-state or $p$-state.

The evaluation of observables from the effective Hamiltonian requires summing over final states for the squared matrix elements. It is therefore crucial to know where the transition strength is concentrated. An important observation simplifies this problem: both the single-particle operator and the momentum transfer are very similar to those in $\mu$ capture and radiative $\pi$ capture. In fact, the single-particle operator is the same axial term $\vec{\sigma}_{i} \tau_{i}$, while the momentum transfer for a pion at rest is in the range $0 \leq \vec{q}^{2} \leq \mathrm{m}_{\pi}^{2}$ with a typical value of $\mathrm{m}_{\pi}^{2} / 2$, i.e. even smaller than in the radiative $\pi$ capture process. There are therefore excellent reasons to believe that the same theoretical methods which are used in the more familiar processes can be applied here with the same level of confidence. As a first step consider the amplitude for the process $\pi^{-}+p \rightarrow n+\gamma_{1}+\gamma_{2}$. We use the notation of Bjorken and Drel1 [7]. In the effective Hamiltonian only the non-relativistic limit will be needed. Consequently we only retain terms which contribute to leading order in the mass ratio $\mathrm{m}_{\pi} / \mathrm{M}$. In addition we take the nucleon to be point-like in view of the low-momentum transfer. of all the possible diagrams required to build a gauge-invariant amplitude, the three important ones are given in Figs. 1a, b, c. In addition there is also a contribution from the process $\pi^{0}+2 \gamma$ following charge exchange on the proton (Fig. 1d), as already discussed by Lapidus [8]. Although necessary for gauge invariance, this graph is easily found to be numerically unimportant in the Coulomb gauge which is used in the following. The important part of the relativistic amplitude is thus: 


$$
\begin{aligned}
& M=\sqrt{2} e^{2} g_{\pi N N} \tau^{-} \bar{u}(x)\left[-r_{5} \frac{x-k_{1}+M}{2 p \cdot k_{1}} \notin_{1} \frac{\pi \cdot \epsilon_{2}}{\pi \cdot k_{2}}-\right. \\
& -\gamma_{5} \frac{k-k_{2}+M}{2+\cdot k_{2}} \epsilon_{2} \frac{\pi \cdot \epsilon_{1}}{\pi \cdot k_{1}}+\left(2 \epsilon_{1} \cdot \epsilon_{2}+\frac{2 \epsilon_{1} \pi \epsilon_{2} \cdot\left(\pi-k_{1}\right)}{\pi \cdot k_{1}}+\right. \\
& \left.\left.+\frac{2 \epsilon_{2} \cdot \pi \epsilon_{1} \cdot\left(\pi-k_{2}\right)}{\pi \cdot k_{2}}\right) \gamma_{5} \frac{1}{-2 k_{1} \cdot k_{2}+2 \pi \cdot\left(k_{1}+k_{2}\right)}\right] u(p)
\end{aligned}
$$

We have here used a pseudo-scalar $\pi N$ vertex with the coupling constant $g_{\pi N N}$. In order to display explicitly the relative $s-$ and $p$-wave $\pi \mathbb{N}$ terms we develop Eq. (1) to linear terms in the pion momentum operator $\vec{\pi}$, which in the nonrelativistic limit gives

$$
\begin{aligned}
& M_{N R}=\frac{\sqrt{2} e^{2}}{2 M} g_{\pi N N} \tau-\left[\vec{\sigma} \cdot \vec{\epsilon} \frac{\vec{\pi} \cdot \vec{\epsilon}_{2}}{m_{\pi} \omega_{1}}+\vec{\sigma} \cdot \vec{\epsilon}_{1} \frac{\vec{\pi} \cdot \vec{\epsilon}_{2}}{m_{\pi} \omega_{2}}+\right. \\
& \left.+\vec{\sigma} \cdot(\vec{\pi}-\vec{\rho}) \frac{\vec{\epsilon}_{1} \cdot \vec{\epsilon}_{2}}{D}-\vec{\sigma} \cdot \vec{\varphi} \frac{\left[\vec{\pi} \cdot \bar{\epsilon}_{1} \hat{k}_{1} \cdot \vec{\epsilon}_{2}+\vec{\pi} \cdot \vec{\epsilon}_{2} \vec{k}_{2} \cdot \vec{\epsilon}_{1}\right]}{m_{\pi} D}\right]
\end{aligned}
$$

where

$$
\begin{aligned}
& D=m_{\pi}\left(\omega_{1}+\omega_{2}\right)-\omega_{1} \omega_{2}(1-y) ; y=\cos \theta_{12} ; \vec{\pi}=-i \vec{\nabla} \\
& \text { and } \vec{\varphi}=\vec{k}_{1}+\vec{k}_{2}
\end{aligned}
$$

It should be noted that the same limit (2) is obtained using a pseudo-vector $\pi N$ coupling obeying PCAC. This is completely analogous to the Kroll-Ruderman limit of ordinary radiative $\pi$ capture: the capture amplitude is independent of the coupling in the low-energy limit.

3. The corresponding effective Hamiltonian is given by

$$
H_{\text {off }}=\sum_{i=1}^{A} M_{N R}(i) e^{-i \vec{\varphi} \cdot \vec{x}_{i}} \Phi_{\pi}\left(\vec{x}_{i}\right) \equiv \sum_{i} O_{i} r_{i}^{-}
$$


This Hamiltonian merits the following comments:

i) The presence of the linear terms in the pion momentum is due to the fact that any light particle radiates easily when its velocity changes.

ii) Meson exchange effects have been neglected. One should note that an important class of meson exchange terms (Fig. le) are suppressed by $\omega_{\gamma} / M$ with respect to the dominant ones. In the case of a single nucleon the principal p-wave $2 \gamma$ term is the Bremsstrahlung of the ordinary radiative $\pi \gamma$ process proportional to $\omega_{\gamma}^{-1}$. This should be compared with the crossed Compton amplitude on two nucleons [9] supplemented by a $\pi$-nucleon vertex which is proportional to $\mathrm{M}^{-1}$. Such meson-exchange terms are therefore small.

iii) The effective Hamiltonian (3) presupposes as all impulse approximations, that the process is "sufficiently local", i.e. confined to a single nucleon. This condition becomes questionable whenever the photon frequencies match nuclear frequencies. In particular, a two-step process in which first a normal radiative capture occurs to an excited state, which subsequently decays by $\gamma$ emission, is a $2 \gamma$-reaction outside our present approach. To minimize such contributions we require the photons to have sufficiently high energy (in practice $\omega_{Y} \geq 10-15 \mathrm{MeV}$ ).

Within these approximations it is straightforward to write the differential $2 \gamma$ capture rate in analogy with the $1 \gamma$ capture rate $[10]$

$$
\frac{d \Lambda^{2 \gamma}}{d \Omega_{12} d \omega_{1}}=\frac{1}{8 m_{\pi}}\left(1+\frac{m_{\pi}}{M}\right)^{2} \frac{1}{2 l+1} \frac{1}{2 J+1} \cdot \sum_{b}\left|\left\langle b\left|H_{+f t}\right| 0\right\rangle\right|^{2} \frac{\omega_{1} \omega_{2}}{(2 \pi)^{4}}
$$

Here $\omega_{2}=m_{\pi}-E-\omega_{1}$ and $\int \mathrm{d} \Omega_{12}=4 \pi$. A factor $1 / 2$ is included, since the two final photons are identical. The transformation from the $\pi N$ to the $\pi$ nucleus c.m. system introduces the kinematical factor $\left(1+m_{\pi} / M\right)^{2}$.

4. Some general. features of the process are apparent even with the oversimplified assumption that the entire transition strength is concentrated to a 
single nuclear excitation energy $\overline{\mathrm{E}}$. If the nucleus has $\mathrm{T}=0$ and if the nuclear interaction potential commutes with the transition operator, it is straightforward to evaluate all physical quantities from the energy weighted sum rule (generalized Thomas-Reiche-Kuhn) [11]. This technique has already been successfully used in the description of $\mu$ capture by Christillin et al. [12] and later extended to single radiative $\pi$ capture by Lipparini et a1. [13]. In view of similarity of momentum transfer and of excitation operators we expect it to work equally well here. The result is simply an expectation value over individual nucleon contributions:

$$
\begin{aligned}
\left.\sum_{b}\left|\left\langle b\left|\sum_{i} O_{i} \tau_{i}\right| 0\right\rangle\right|^{2}=(2 \bar{E})^{-1}\langle 0| \sum_{i} \frac{\left(\vec{\nabla} O_{i}\right.}{2 M}\right)^{2}|0\rangle= \\
=\frac{Z}{2 \overline{E M}}\left(S\left|\Phi_{\pi}\right|^{2}+P\left|\vec{\nabla} \Phi_{\pi}\right|^{2}\right) \cdot\left(\frac{\sqrt{2} t^{2}}{2 M} g_{\pi N N}\right)^{2} .
\end{aligned}
$$

where $\left|\phi_{\pi}\right|^{2}$ and $\left|\vec{\nabla} \phi_{\pi}\right|^{2}$ axe averages over the nuclear density and with the explicit form of the coefficients $S$ and $P$ given below.

$$
\begin{aligned}
S= & \frac{Q^{4}\left(1+y^{2}\right)}{D^{2}} \\
P= & \frac{4}{3}\left(\frac{1}{\omega_{1}^{2}}+\frac{1}{\omega_{2}^{2}}+\frac{1+y^{2}}{2 \omega_{1} \omega_{2}}\right) \frac{Q^{2}}{m_{\pi}^{2}}+ \\
& +\frac{2}{3} \frac{1}{D}\left[\frac{Q^{2}}{m_{\pi}}\left(\frac{1}{\omega_{1}}+\frac{1}{\omega_{2}}\right)\left(1+y^{2}\right)-y\left(1-y^{2}\right)-\frac{2 Q^{2}}{m_{\pi}^{2}}(2-y)\left(1-y^{2}\right)\right] \\
& +\frac{2}{3} \frac{Q^{2}}{D^{2}}\left[4\left(1+y^{2}\right)+2 y\left(1-y^{2}\right)+\frac{Q^{2}}{m_{x}^{2}}(2-y)\left(1-y^{2}\right)\right]
\end{aligned}
$$

We note that the average over all directions for stopping pions has eliminated all interference terms. A first application of the result gives approximate expressions of the doubly radiative rate in closed form. For simplicity we restrict the discussion to light nuclei for which capture takes place from 1 s and $2 p$ atomic states with wave functions which are constant and linear over the nuclear volume: 


$$
\begin{aligned}
& \frac{d \Lambda_{1 S}^{2 \gamma}}{d \Omega_{12}}=\frac{1}{8 m_{\pi}}\left(1+\frac{m_{\pi}}{M}\right)^{2} \frac{1}{2 J+1} \frac{z \alpha^{2} g_{\pi N N}^{2}}{(2 \pi)^{2} M^{3} \bar{E}}\left|\Phi_{\pi}\right|^{2} \int_{\Delta}^{m_{\pi}-\bar{E}-\Delta} d \omega_{1} \omega_{1} \omega_{2} S \\
& \frac{d \Lambda_{2 r}^{2 \gamma}}{d \Omega_{12}}=\frac{1}{8 m_{\pi}}\left(1+\frac{m_{\pi}}{M}\right)^{2} \frac{1}{2 J+1} \frac{z \alpha^{2} g_{\pi N N}^{2}}{(2 \pi)^{2} M^{3} \bar{E}} \frac{\left|\vec{\nabla} \Phi_{\pi}\right|^{2}}{3} \int_{\Delta \pi}^{m_{\pi}-\bar{E}-\Delta} d \omega_{1} \omega_{1} \omega_{2}\left(P+S \frac{\left.r^{2}\right\rangle}{3}\right)
\end{aligned}
$$

where $\Delta$ is the experimental energy cut-off.

Inspection of Eqs. (7) reveals that the is capture is exclusively given by the $\left(\pi^{-} \pi^{+}\right)$annihilation graph to the order considered. A more careful examination of Eq. (7b) shows that the $2 p$ capture is numerically dominated by the momentumdependent Bremsstrahlung contributions to $P$.

From the expressions (6) and (7) we can directly read of the shape of the angular correlations: they are dominated by the factor $\left(\vec{k}_{1}+\vec{k}_{2}\right)^{2}$, indicating a decreasing angular distribution with increasing angle between the photons. This basic angular distribution is somewhat modified by a slowly varying function of the angle. This conclusion is of course not dependent on the use of an energyweighted sum rule. Alternatively, a naive Fermi gas model leads to a smaller rate in the backward direction, simply because the low-momentum transfer displaces the Fermi sphere comparatively little into the region allowed by the exclusion principle.

From the approximate expressions (6) and (7) one can also see the general features of the energy distributions. The capture from the $2 p$ state has the characteristic Bremsstrahlung shape with denominators $\omega_{1}^{-1}$ and $\omega_{2}^{-1}$, diverging for sma11 $w_{i}$. The energy distribution has a minimum for equipartition. The total rate from this state is dependent on the low-energy cut-off with a logarithmic singularity. On the contrary, the annihilation from the 1 s state has a maximum for equipartition and a total rate that is independent of the low-energy cut-off. 
5. For a more precise discussion we now specialize to ${ }^{12} \mathrm{C}$, for which some experimental data are available [5]. The branching ratio $R_{2 \gamma}$ is given by

$$
R^{2 \gamma}=\left(\frac{\Lambda^{2 \gamma}}{\lambda_{a}}\right)_{1 s} \omega_{s}+\left(\frac{\Lambda^{2 \gamma}}{\lambda_{a}}\right)_{2 r} \omega_{r}
$$

Here $\omega_{s}=0.08$ and $\omega_{p}=0.92[10]$ are the probabilities that a pion is absorbed from the $1 s$ and the $2 p$ orbit in the atomic cascade. The normal fate of a pion stopping in ${ }^{12} \mathrm{C}$ is therefore absorption from the $2 \mathrm{p}$ state. The total absorption rates $\lambda_{a}(1 s)=3 \mathrm{keV}$ and $\lambda_{a}(2 \mathrm{p})=2 \mathrm{eV}$ from these two states will be used $[10]$. Moreover, since $\mathrm{R}^{2 \gamma}$ depends on the experimental cut-offs, we will use those in the recent experimental set-up, $\omega_{1}, \omega_{2} \geq 25 \mathrm{MeV}, \theta_{12} \geq 42^{\circ}[5]$. Predictions below therefore specifically refer to these experimental data.

The averages of the atomic wave functions over the nucleus

$$
\begin{aligned}
\left|\phi_{15}\right|^{2} & =\frac{\left(z \alpha m_{\pi}\right)^{3}}{\pi} C_{15} \\
\frac{1}{3}\left|\vec{\nabla} \phi_{2 \mu}\right|^{2} & =\frac{\left(z \alpha m_{\pi}\right)^{5}}{32 \pi} C_{2 \mu}
\end{aligned}
$$

have the nuclear distortion factors $C_{1 s}=0.5$ and $C_{2 p}=1.4[10]$ with respect to the point hydrogenic values. The ${ }^{12} \mathrm{C} \mathrm{r} . \mathrm{m} . \mathrm{s}$. radius is $\left\langle\mathrm{r}^{2}\right\rangle^{1 / 2}=2.45 \mathrm{fm}[14]$. With these values it is now straightforward to see that the ${ }^{12} \mathrm{C}\left(\pi^{-}, 2 \gamma\right)$ process is dominated by the capture from the $2 \mathrm{p}$ state. The nucleus ${ }^{12} \mathrm{C}$ differs from the previously discussed case in so far as it is not a spin-saturated structure. As a consequence there is even at zero momentum transfer a super allowed axial transition between the ${ }^{12} \mathrm{C}$ ground state $\left(J=0^{+} ; \mathrm{T}=0\right)$ and the ${ }^{12} \mathrm{~B}$ ground state $\left(\mathrm{J}=1^{+} ; \mathrm{T}=1\right)$. This transition between two we11-known nuclear states has been calculated using Eq. (3). The result has been added to the previous sum rule (7), in which it is not included, since its derivation implicitly assumes exact SU(4) symmetry in view of the spin dependence of the transition operator. It gives rise 
to a nearly isotropic angular correlation between the two photons, adding in as a "background" term to the previously discussed shape (see Fig. 2a).

A first estimate is obtained using the approximation of a unique nuclear excitation energy $\bar{E}$ as in the previous section. From an inspection of the excitation energy spectrum in ${ }^{12} \mathrm{C}\left(\pi^{-}, \gamma\right){ }^{12} \mathrm{~B}^{*}$ as well as from the shape of the nuclear photo absorption cross-section one finds an average excitation energy $\overline{\mathrm{E}} \simeq 23 \mathrm{MeV}$. The angular distribution is shown in Fig. $2 a$ and $\mathrm{R}^{2 \gamma}$ turns out to be $1.1 \times 10^{-5}$. The corresponding branching ratio for single radiative capture, where the Hamiltonian is consistently taken to be

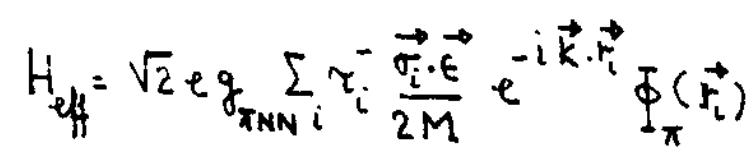

turns out to be $\mathrm{R}^{1 Y}=2.15 \%$ to be compared with $\mathrm{R}_{\exp }^{1 Y}=1.92 \pm 0.2 \%$. This represents a good over-all check on out procedure.

An improved treatment can be obtained as follows. Apart from the allowed transition, the excitation spectrum at low momentum transfer will be dominated by the axial giant dipole states, which coincide in energy with that of the usual giant dipole one if the nuclear forces are spin-isospin independent [SU(4) symmetry]. With increasing momentum transfer this transition is depleted in favour of higher multipole ones to higher excitation. We approximate these features phenomenologicaliy for moderate momentum transfers by two Lorenz curves for the different types of transitions, each exhausting a classical sum rule. The energy and width of the unretarded dipole excitation are taken from nuclear photoreactions as before: $E_{1}=23 \mathrm{MeV}, \Gamma_{1}=5 \mathrm{MeV}$. The parameters of the higher multipole transition $E_{2}=35 \mathrm{MeV}, \Gamma_{2}=12 \mathrm{MeV}$, are adjusted to the observed ${ }^{12} \mathrm{C}\left(\pi^{-}, \gamma\right)^{12} \mathrm{~B}^{*}$ excitation spectrum $[10]$.

In the spirit of Foldy and Walecka [15], the strength of the first resonance is multiplied by the square of the elastic form factor of the ${ }^{12} \mathrm{C}$ ground state $\left|F e\left(\vec{q}^{2}\right)\right|^{2}$, which incorporates the quenching effect of retardation on the 
"unretarded" giant resonance. The second resonance is then assumed to be affected by a corresponding factor $1-\left|F_{e l}\left(\overrightarrow{\mathrm{q}}^{2}\right)\right|^{2}$. Finally the excitation spectrum is normalized so as to reproduce the rate obtained using closure at the momentum transfer of single radiative capture.

The nuclear response $R\left(E, \vec{q}^{2}\right)$ is therefore given by a family of curves in the energy-momentum transfer plane. In this_case the previous formulae (7) are easily modified by the transformation $\int_{\Delta}^{m_{\pi}-\bar{E}-\Delta} d \omega_{1} \frac{1}{E} \rightarrow \int_{\Delta}^{m_{\pi}-\Delta} d w_{1} \int_{0}^{m_{\pi}-\Delta-\omega_{1}} \frac{d E}{E} R\left(E, \vec{q}^{2}\right)$ Since the closure approximation is no longer made, certain physical quantities, like the photon energy distributions, can now be predicted more realistical1y.

The ${ }^{12} \mathrm{C}$ branching ratio $\mathrm{R}^{2 \gamma}$ is now $0.9 \times 10^{-5}$. The principal difference from the closure result is a weak additional $\vec{q}^{2}$ dependence of the mean excitation energy, as is seen from the angular distribution (fig. 2b).

In conclusion we regard the predictions of the present approach as our final result. A reasonable hint about the model (in)dependence can be obtained from the comparison with the results of the closure approximation which has a strongly dissimilar spectrum of excitation energies. The cut-off dependence of the results is displayed in $\mathrm{fig} .2 \mathrm{c}$.

In $\mathrm{fig}$. 2b we have also given the contribution from the annihilation graph separately, since this is the term expected to be particularly sensitive to anomalies in the nuclear meson structure. One notes that it is generally unimportant, and even in the background direction its contribution is not dominant. This is in disagreement with the conclusion of ref. 3 .

In the same figure the present experimental data [5] are also given. One notes, that although the total rate is reproduced, the measured values have an angular correlation rising in the backward direction, contrary to the general theoretical prediction of a conventional nuclear model. 
6. We have thus developed a quantitative theory for the $\left(\pi^{-}, 2 \gamma\right)$ capture in nuclei, assuming a conventional nucleus without anomalous meson structure. As an application we have computed the various properties of the capture reaction for the particular case of ${ }^{12} \mathrm{C}$. In this nucleus the capture occurs predominantly from the atomic $2 p$ state. Using the restriction given by the experimental acceptance we find that the rate is in essential agreement with experimental data. On the contrary, there is a discrepancy in the angular distribution in the backward direction. If this anomaly is confirmed, it indicates that some novel feature must be added to the theoretical picture. One possibility would be a preferential enhancement in the backward direction of the pion "annihilation process". However, one should be aware that present momentum transfers are smaller than those for which an important enhancement might be expected as a precursor effect to pion condensation $\left(|\vec{q}| \simeq 2 \mathrm{fm}^{-1}\right)[4]$. Within a conventional nuclear picture the theoretical situation is by now sufficiently clear.

It would be welcome to have the experimental situation clarified beyond doubts and to have further experiments in other nuclei.

\section{Acknowledgements}

We have profited from interesting remarks by Professors J. Deutsch and M. Ericson. 


\section{REFERENCES}

1) T.E.0. Ericson and C. Wilkin, Phys. Lett. 57B, 345 (1975).

2) A.B. Migdal, Sov. Phys. JETP 34, 1184 (1972),

3) S. Barshay, Phys. Letters $\underline{78 B}, 384$ (1978) and $\underline{79 B}, 514$ (1978).

4) J. Delorme and M. Ericson, Phys. Letters 76B, 182 (1978).

5) J. Deutsch et a1., Phys. Letters $80 \mathrm{~B}, 347$ (1979).

6) D. Beder, $\pi^{-} p \rightarrow \gamma \gamma n$ at threshold, Univ. of British Columbia preprint, November 1978 .

7) J.D. Bjorken and S.D. Dre11, Relativistic quantum mechanics (McGraw Hill, New York, 1964).

8) L.I. Lapidus and M.M. Musakhanov, Sov. J. Nuc1. Phys. 15, 558 (1972).

9) P. Christillin and M. Rosa Clot, Nuovo Cimento 28A, 29 (1975).

10) H.W. Baer et al., Adv. Nuc1. Phys. 9, 177 (1977).

11) See, for example, T.E.0. Ericson and M.P. Locher, Nuc1. Phys. A148, 1 (1970).

12) P. Christillin, A. Dellafiore and M. Rosa Clot, Phys. Rev. Lett. 31, 1012 (1973).

13) E. Lipparini, S. Stringari and M. Traini, Nuovo Cimento 34A, 48 (1976).

14) C.W. De Jager, H. De Vries and C. De Vries, At. Data and Nuc1. Data Tables 14, 479 (1974).

15) L.L. Foldy and J.D. Walecka, Nuovo Cimento $\underline{34}, 1026$ (1964). 


\section{Figure captions}

Fig. 1 : Relevant graphs in doubly radiative $\pi$ capture.

Fig. 2 : Theoretical angular distributions of the branching ratio for ${ }^{12} \mathrm{C}$ as a function of $y=\cos \theta_{12}(\Delta=25 \mathrm{MeV}$; for definitions see text).

a) : (-) sum rule, (-) integral over the excitation spectrum, without allowed transitions, (-...-) allowed transitions only.

b) : (-) final result (integral over the excitation spectrum + allowed contributions), (-) contributions of the annihilation graph only. The average momentum transfer $\sqrt{\left\langle\mathrm{q}^{2}\right\rangle}$ at each angle is indicated at the top of the figure in MeV/c. i shows the experimental data of Ref. 5 .

c) : Predicted angular distribution for different values of the cut-off. $(--) \Delta=15 \mathrm{MeV},(-\cdot) \Delta=35 \mathrm{MeV}$. 


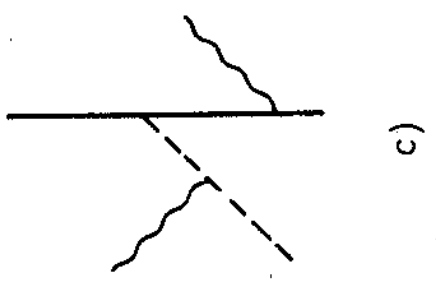

$+$
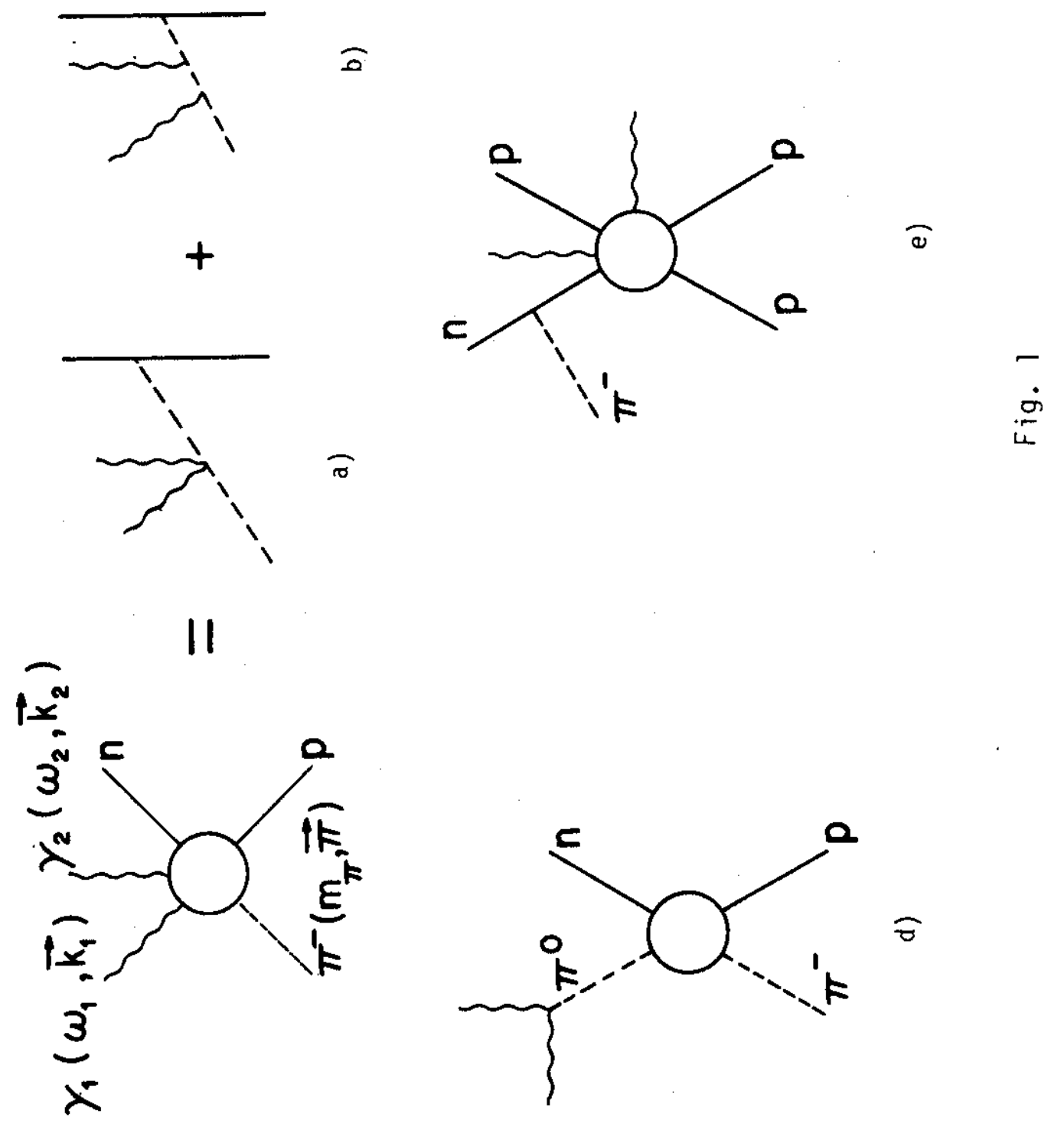


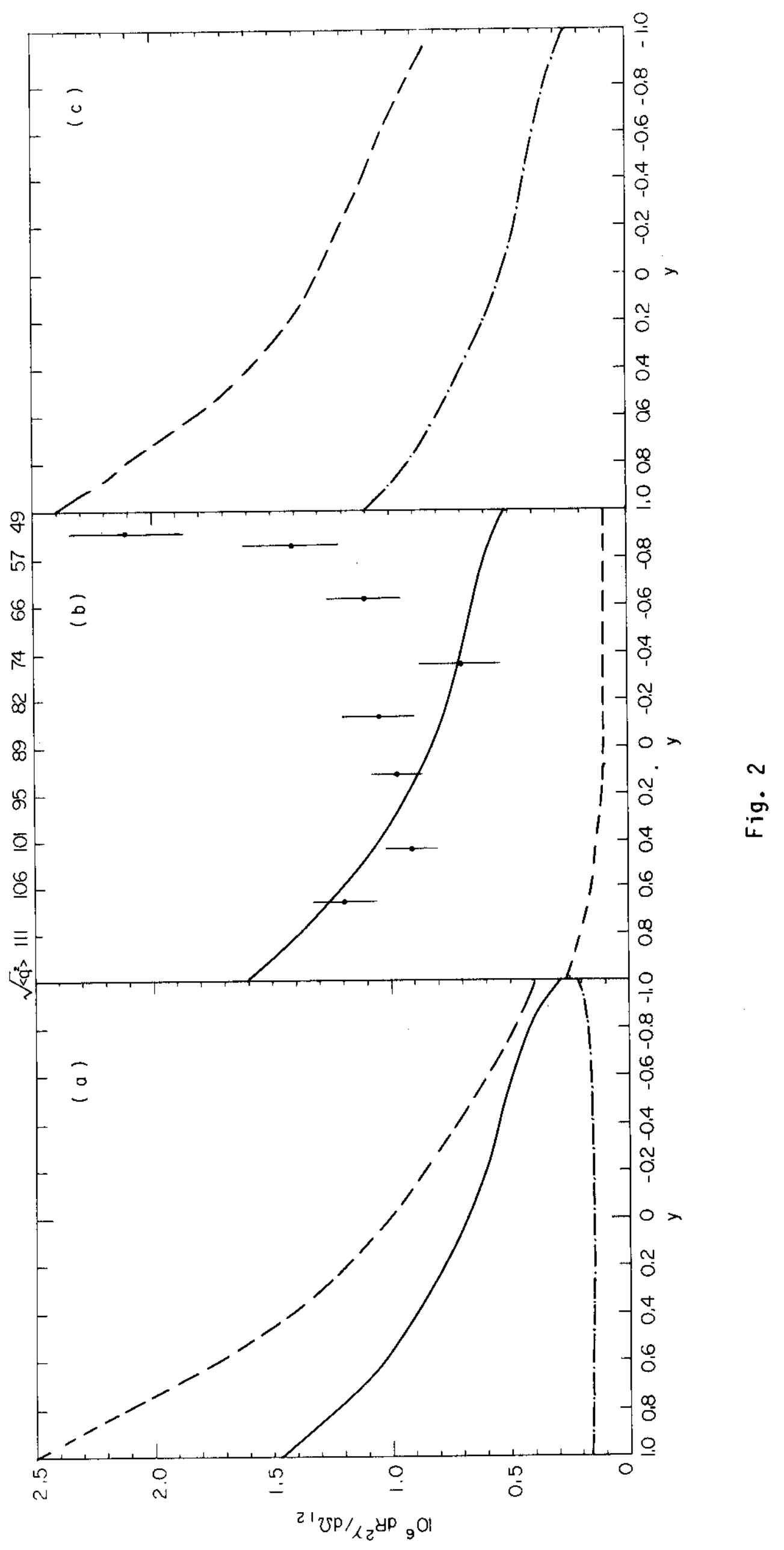

